\title{
TRIM45 functions as a tumor suppressor in the brain via its E3 ligase activity by stabilizing p53 through K63-linked ubiquitination
}

\author{
Jindong Zhang ${ }^{1,2,6}$, Chuanxia Zhang ${ }^{3,6}$, Jun Cui ${ }^{\star, 2,3,6}$, Jiayu Ou ${ }^{1}$, Jing Han ${ }^{1}$, Yunfei Qin ${ }^{3}$, Feng Zhi ${ }^{\star *, 4}$ and Rong-Fu Wang ${ }^{\star, 5}$
}

Tripartite motif-containing protein 45 (TRIM45) belongs to a large family of RING-finger-containing E3 ligases, which are highly expressed in the brain. However, little is known regarding the role of TRIM45 in cancer biology, especially in human glioma. Here, we report that TRIM45 expression is significantly reduced in glioma tissue samples. Overexpression of TRIM45 suppresses proliferation and tumorigenicity in glioblastoma cells in vitro and in vivo. In addition, CRISPR/Cas9-mediated knockout of TRIM45 promotes proliferation and inhibits apoptosis in glioblastoma cells. Further mechanistic analyses show that TRIM45 interacts with and stabilizes p53. TRIM45 conjugates K63-linked polyubiquitin chain to the C-terminal six lysine residues of p53, thereby inhibiting the availability of these residues to the K48-linked polyubiquitination that targets p53 for degradation. These findings suggest that TRIM45 is a novel tumor suppressor that stabilizes and activates p53 in glioma.

Cell Death and Disease (2017) 8, e2831; doi:10.1038/cddis.2017.149; published online 25 May 2017

Gliomas are the most common type of brain tumors and account for $\sim 80 \%$ of primary malignant tumors in the central nervous system (CNS). ${ }^{1-3}$ Currently, the standard treatment strategies for glioma are surgical resection and adjuvant chemotherapy with temozolomide (TMZ) combined with radiotherapy. ${ }^{4}$ However, patients with malignant glioma, especially those with glioblastoma (GBM), have little benefit from standard treatments, due to the tumor's malignant features such as rapid cell proliferation, robust invasiveness, and increased angiogenesis, and lack of specific therapeutic targets for immunotherapy. ${ }^{1,5}$ Therefore, it is imperative to determine the mechanisms underlying glioma tumorigenesis and to develop more effective therapeutic strategies.

The tumor suppressor p53 is a crucial cellular stress sensor that triggers cell-cycle arrest, apoptosis, and senescence in response to many diverse stresses, including DNA damage, hyperproliferative signals, hypoxia, oxidative stress, ribonucleotide depletion, and nutrient starvation. ${ }^{6}$ p53-mediated cellular responses primarily depend on the function of p53 as a transcriptional activator and on the p53-mediated induction of particular target genes. ${ }^{6}$ As even minimal changes in p53 expression can be deleterious to the organism, tight regulation of p53 is essential in normal cells..$^{7-9}$ A plethora of redundant post-transcriptional modifications can occur in p53, including ubiquitination, acetylation, phosphorylation, methylation, neddylation, and sumolytion, all of which significantly affect its activity and function. ${ }^{7}$

Ubiquitination is a reversible post-translational modification that either targets proteins for degradation or regulates protein function. ${ }^{10}$ Ubiquitin itself contains seven lysines, and each of these can be further conjugated to another ubiquitin molecule at its carboxyl terminus to form different types of polyubiquitin chains. The lysine-(K) 48- and K63-linked polyubiquitin chains are the predominant types of ubiquitin linkage. ${ }^{11} \mathrm{~K} 48$-linked polyubiquitination targets proteins for proteasomal degradation, and K63-linked polyubiquitination has a role in cell signaling. The effects and mechanisms of several E3 ligases on p53 have been reported. ${ }^{12}$ The RING-finger E3 ligase mouse double minute homolog 2 (MDM2) conjugates K48linked polyubiquitin chains to $\mathrm{p} 53$, and this modification targets p53 for proteasomal degradation. ${ }^{13,14}$

The tripartite motif-containing (TRIM) family is characterized by several unique structural motifs: a RING-finger domain, one or two B-box domain and a coiled-coil domain. ${ }^{15,16}$ Most TRIM proteins possess a variable $\mathrm{C}$-terminal domain, which has a role in substrate binding. ${ }^{17}$ As a member of the TRIM family, TRIM45 contains a filamin-type immunoglobulin (FLMN) domain in its carboxy-terminal region. TRIM45 was reported to negatively regulate the MAPK signaling pathway by inhibiting RACK1/PKC complex formation. ${ }^{18,19}$ It also negatively regulates NF-kB signaling. ${ }^{20}$ Although TRIM45 mRNA

\footnotetext{
${ }^{1}$ Zhongshan School of Medicine, Sun Yat-sen University, Guangzhou 510080, People's Republic of China; ${ }^{2}$ Collaborative Innovation Center of Cancer Medicine, Sun Yatsen University, Guangzhou 510080, People's Republic of China; ${ }^{3}$ Key Laboratory of Gene Engineering of the Ministry of Education, State Key Laboratory of Biocontrol, School of Life Sciences, Sun Yat-sen University, Guangzhou 510006, People's Republic of China; ${ }^{4}$ Modern Medical Research Center, Third Affiliated Hospital of Soochow University, Changzhou 213000, People's Republic of China and ${ }^{5}$ Center for Inflammation and Epigenetics, Houston Methodist Research Institute, 6670 Betner Avenue, Houston, TX 77096, USA

${ }^{*}$ Corresponding author: J Cui, Collaborative Innovation Center of Cancer Medicine, Sun Yat-sen University, Eastern Campus, Room 320, Building of School of Life Science, Sun Yat-sen University, Guangzhou 510006, People's Republic of China. Tel: +86 20 39943429; E-mail: cuij5 @ mail.sysu.edu.cn or F Zhi, Modern Medical Research Center, Third Affiliated Hospital of Soochow University, No. 185 Juqian Road, Changzhou 213000, People's Republic of China. Tel: +86 519 688708 99; E-mail: danielzhif@ 163.com or R-F Wang, Center for Inflammation and Epigenetics, Houston Methodist Research Institute, 6670 Betner Avenue, Houston, TX 77096, USA. Tel: +1 713 441 7359; Fax: +713 441 5439; E-mail: rwang3@ @oustonmethodist.org

${ }^{6}$ These authors contributed equally to this work.

Received 25.8.16; revised 10.1.17; accepted 13.2.17; Edited by Y Haupt
} 


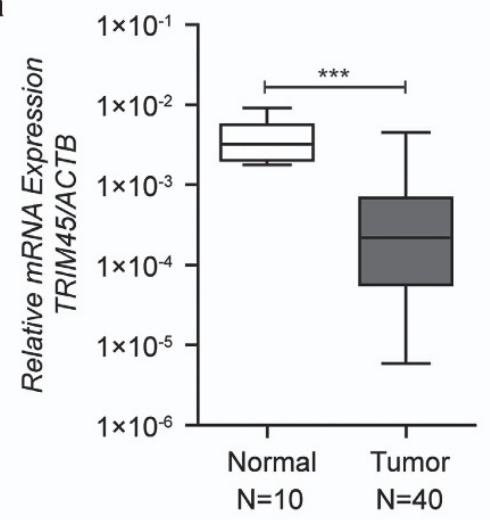

b

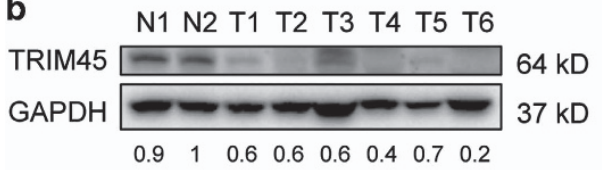

C

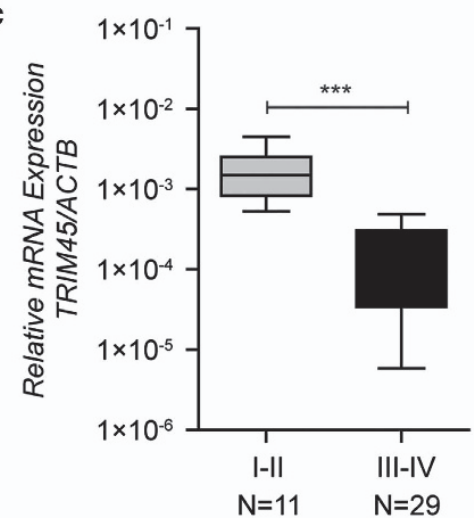

d
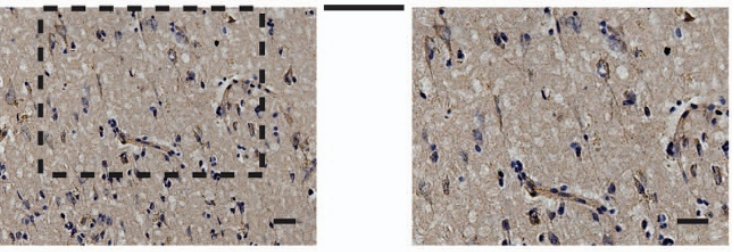

Grade 1
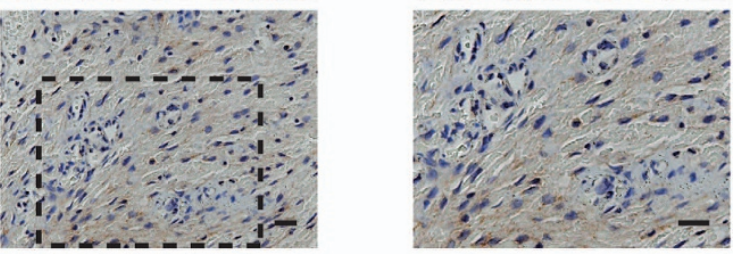

Grade 2
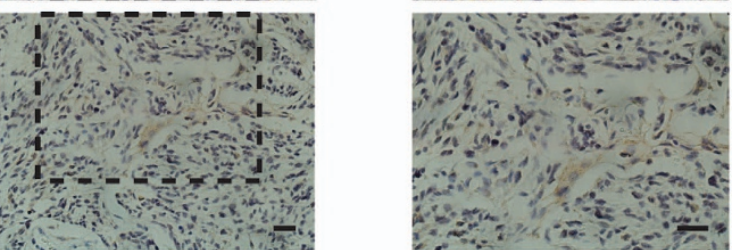

Grade 3
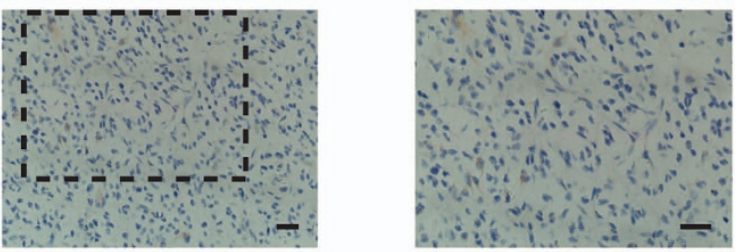

Grade 4
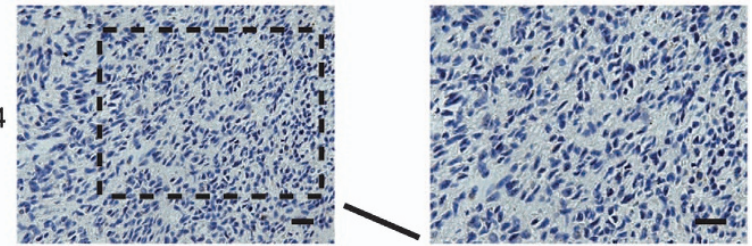

Figure 1 TRIM45 expression is reduced in glioma tissues. (a) TRIM45 mRNA levels in normal brain tissues (Normal) and glioma tissues (Tumor) were determined by realtime PCR. (b) Representative images from immunoblot analyses of TRIM45 levels in normal brain tissues (N) and glioma tissues (T). Protein expression levels were normalized to glyceraldehyde 3-phosphate dehydrogenase (GAPDH) levels. (c) Comparison of TRIM45 mRNA levels among gliomas of different pathological grades. (d) Representative images from immunohistochemistry (IHC) assays of paraffin-embedded specimens. Scale bars: $100 \mu \mathrm{m}$. Data in panels (a and $\mathbf{c}$ ) are presented as the mean \pm S.D. ${ }^{* \star *} P<0.001$

is highly expressed in the brain of human adult and embryonic tissues, ${ }^{18}$ its function in primary CNS tumors has not been investigated yet.

Here we report that TRIM45 expression is reduced in glioma tissues, and TRIM45 suppresses proliferation and tumorigenicity in GBM cells in vivo and in vitro. Furthermore, TRIM45 interacts with and stabilizes p53 by promoting K63-linked polyubiquitination of $\mathrm{p} 53$, thereby inhibiting the subsequent K48-linked polyubiquitination that targets p53 for degradation. These findings suggest that TRIM45 functions as a novel regulator responsible for maintaining p53 stability in glioma.

\section{Results}

TRIM45 is downregulated in primary gliomas. To determine the functional and clinical relevance of TRIM45 in human glioma, we first examined TRIM45 expression levels in normal brain tissues and human primary glioma tissues using quantitative real-time PCR (qRT-PCR) and immunoblot assays. As shown in Figure 1a, TRIM45 mRNA was significantly downregulated in glioma samples compared with normal brain tissue samples. Similar results were obtained from immunoblotting experiments, showing the downregulation of TRIM45 protein expression in tumor tissues (Figure 1b). We subsequently investigated whether TRIM45 expression levels represented a distinct molecular signature for a subset of gliomas. TRIM45 mRNA levels were lower in high-grade (WHO grade III/IV) gliomas compared with lowgrade (WHO grade I/II) gliomas (Figure 1c). The reduced expression of TRIM45 in glioma was further confirmed by immunohistochemistry staining of normal brain tissue sections and tumor tissue sections (Figure 1d). Taken together, these data indicated that TRIM45 expression was downregulated in human glioma tissues.

TRIM45 regulates tumor cell growth in vitro and in vivo. To investigate the function of TRIM45 in the progression of glioma, we established U87 MG (referred to as U87) and LN229 glioma cell lines stably overexpressing 
a

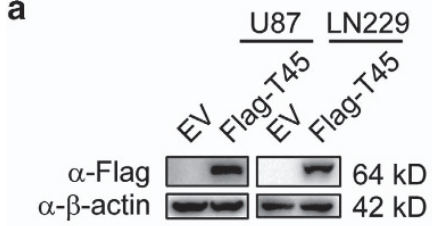

C

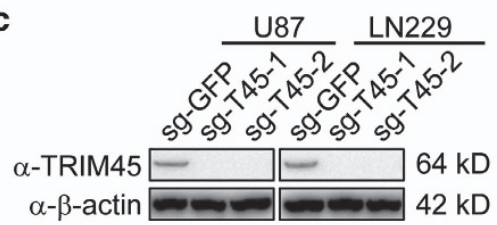

e

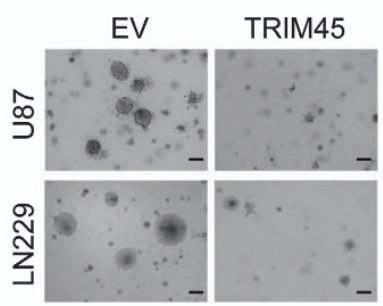

f

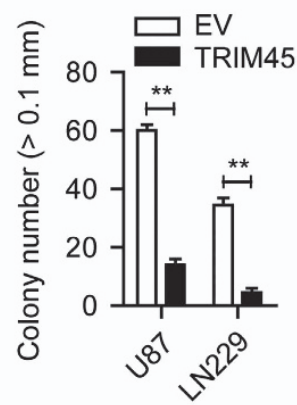

i

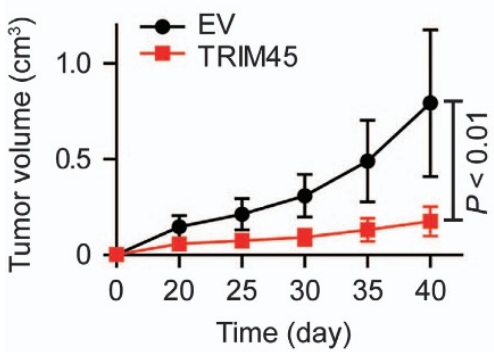

b

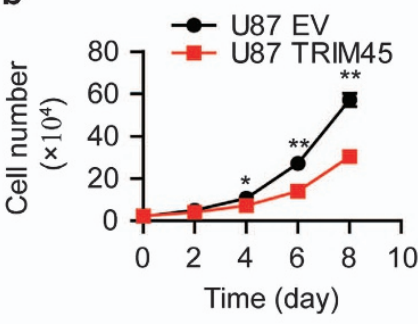

d

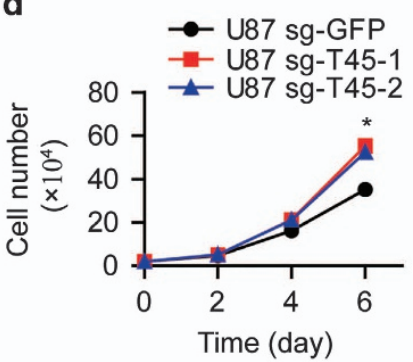

g

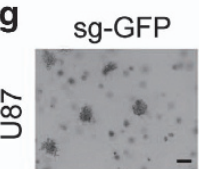

sg-T45-1

$\mathrm{sg}-\mathrm{T} 45-2$

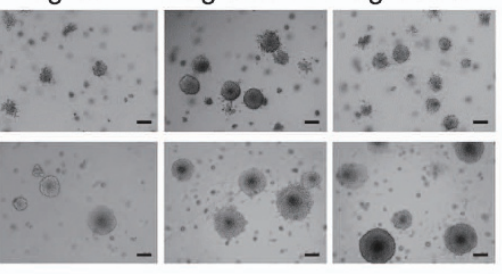

ิㅗㄱ
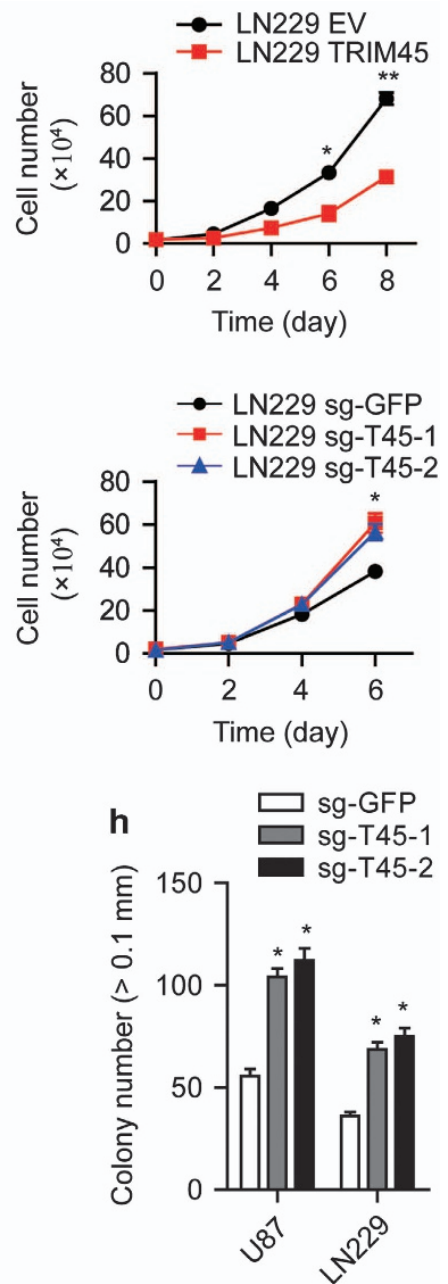

k

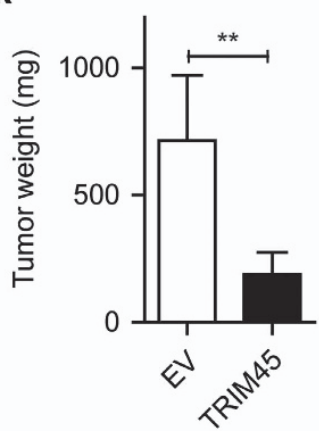

Figure 2 TRIM45 inhibits glioma progression in vitro and in vivo. (a) Lysates of TRIM45-expressing and control U87 and LN229 cells were immunoblotted with anti-Flag antibody. $\beta$-Actin was used as a loading control. (c) Lysates of TRIM45 KO and control U87 and LN229 cells were immunoblotted with an anti-TRIM45 antibody. $\beta$-Actin was used as a loading control. (b and d) Growth curves were performed by plating a fixed number of cells in triplicate and counting cells at different time points. (e-h) Anchorageindependent growth assay of TRIM45-overexpressing (e and f) and TRIM45 KO ( $\mathbf{g}$ and $\mathbf{~ h}$ ) U87 and LN229 cells. Scale bars: $200 \mu \mathrm{m}$. (i-k) Xenograft tumors derived from LN229 cells transfected with TRIM45 or empty vector control lentivirus. Tumor growth (i), representative images of tumor growth (j) and the measured tumor weights (k) are presented. Each bar represents the mean \pm S.D. of three independent experiments. ${ }^{*} P<0.05$ and ${ }^{* *} P<0.01$

Flag-tagged TRIM45 (Figure 2a). The protein level of TRIM45 in TRIM45-expressing U87 and LN229 cells was comparable to that in primary normal human astrocytes (Supplementary Figure 1). The proliferation rate was significantly inhibited in TRIM45-overexpressing U87 and LN229 cells compared with control cells (Figure 2b), whereas TRIM45 knockdown by small hairpin RNA (shRNA) increased the proliferation rate (Supplementary Figures $2 \mathrm{~A}$ and $\mathrm{B}$ ). To further confirm these findings, we used the CRISPR/Cas9 system to genetically knockout (KO) the TRIM45 gene in U87 and LN229 cells 
using two independent single-guide RNAs (gRNAs) targeting TRIM45 (Figure 2c and Supplementary Figure 3). We found that TRIM45 KO led to accelerated proliferation in both U87 and LN229 cells (Figure 2d). We next performed a colony formation assay in soft agar to evaluate the effect of TRIM45 on anchorage-independent growth in glioma cells. Notably, overexpression of TRIM45 significantly inhibited the number and size of the colonies in both U87 and LN229 cells, whereas $\mathrm{KO}$ of TRIM45 exerted the opposite effect (Figures 2e-h). Similar results were observed in TRIM45knockdown glioma cells (Supplementary Figure 4). These results suggest that TRIM45 has an important role in the tumorigenicity of GBM cells in vitro.

We next evaluated the effects of TRIM45 on tumorigenicity using xenografts mouse model. TRIM45-overexpressing tumors grew at a significantly slower rate compared with control tumors (Figure 2i). The tumors derived from TRIM45overexpressing cells were smaller and weighed less than the control tumors (Figures 2j and k). Taken together, these data indicate that TRIM45 inhibits GBM cell growth in vitro and in vivo.

TRIM45 promotes apoptosis in GBM cells. To further investigate the mechanism of TRIM45-mediated inhibition of GBM cell growth, we evaluated cell-cycle progression and apoptosis in GBM cells. Flow cytometry analysis demonstrated that overexpression of TRIM45 had little effect on cellcycle distribution (Supplementary Figure 5). Annexin V flow cytometric analysis showed that TRIM45 overexpression markedly enhanced apoptosis (Figure 3a), and knockdown or KO of TRIM45 inhibited apoptosis in both U87 and LN229 cells (Figure $3 \mathrm{~b}$ and Supplementary Figure 6A). Furthermore, overexpression of TRIM45 led to more cleaved caspase-3 (Figure 3c), whereas knockdown and KO of TRIM45 led to less cleaved caspase-3 in both U87 and LN229 cells (Figure 3d and Supplementary Figure 6B). Taken together, these results suggest that TRIM45 inhibits the growth of GBM cells by promoting apoptosis.

TRIM45 activates p53 signaling in GBM cells. We next examined the signaling pathway by which TRIM45 inhibits proliferation and induces the apoptosis of GBM cells. As PI3K-Akt and Ras-MAPK signaling pathway are the most frequently activated signaling pathways involved in gliomagenesis and TRIM45 was also reported to be associated with PKC-mediated modulation of the ERK-JNK and NF-KB signaling pathway, ${ }^{19,20}$ we examined the phosphorylation status of AKT, ERK, JNK, p38, and IKK $\beta$ in TRIM45overexpressing or TRIM45 KO glioma cells. We did not detect any change in the phosphorylation status of all the kinases examined, regardless of whether the expression of TRIM45 was upregulated or downregulated (Supplementary Figures 7A and B), suggesting that TRIM45 does not affect the PI3K/AKT, MAPK, and NF-KB signaling pathways in glioma cells.

As the p53 tumor suppressor pathway is one of the most significant pathway implicated in glioma and modulates multiple cellular processes, ${ }^{21,22}$ we assessed levels of p53 in TRIM45-overexpressing and $\mathrm{KO}$ cells, respectively. Overexpression of TRIM45 increased the protein levels of p53 and the p53 target gene PUMA in U87 and LN229 cells (Figure 4a). Conversely, TRIM45 KO by two independent sgRNAs decreased the protein levels of p53 and PUMA in U87 and LN229 cells (Figure 4b). We further detected the differential expression of several p53 target genes using qRT-PCR in TRIM45-overexpressing cells and control cells, and we found that TRIM45 overexpression resulted in much higher expression of p53 target genes, including IGFBP5, TRIM22, IGFBP2, ZMAT1, RPRM, IGFBP3, BBC3 (which encodes PUMA), PMAIP1 (which encodes NOXA), and CDKN1A (which encodes p21) (Figure 4c). Consistently, the mRNA levels of these genes were significantly downregulated in TRIM45 KO cells (Figure 4d). These findings strongly suggest that TRIM45 promotes the transcriptional activity of p53. To test this hypothesis, we evaluated the ability of TRIM45 to influence p53 reporter activity and found that TRIM45 significantly promoted the transcriptional activity of p53 in U87 cells (Figure 4e). Taken together, these results indicated that TRIM45 activates the p53 signaling pathway in GBM cells.

To determine whether TRIM45 affects apoptosis of glioma cells through p53, we knocked down p53 in TRIM45overexpressing and control cells and examined the effect of silencing p53 on TRIM45-mediated apoptosis under TMZ treatment or without treatment (Supplementary Figure 8A). Apoptosis induced by TRIM45 overexpression was reversed by p53 knockdown in untreated U87 cells (Supplementary Figure 8B, left). Furthermore, TRIM45 could not enhance the TMZ-induced apoptosis in p53-knockdown U87 cells (Supplementary Figure 8B, right). These results suggest that apoptosis induced by TRIM45 is p53-dependent.

TRIM45 stabilizes p53. To further determine if TRIM45 regulates the stability of endogenous p53, we measured the half-life of p53 in the presence of cycloheximide (CHX), an inhibitor of protein synthesis. The half-life of p53 decreased in TRIM45 KO U87 cells, indicating that TRIM45 stabilizes endogenous p53 (Figures $4 \mathrm{f}$ and $\mathrm{g}$ ). To exclude the possibility that the change in p53 protein levels occurred at the transcriptional level, we performed RT-PCR using the same glioma cells, and found that p53 mRNA level was not influenced by disruptions of TRIM45 expression (Supplementary Figure 9). The loss of p53 induced by TRIM45 KO was rescued by the proteasome inhibitor MG132 (Figures $4 \mathrm{~h}$ and $\mathrm{i}$ ), indicating that TRIM45 protects p53 from proteasomal degradation.

TRIM45 interacts with p53. The results presented in Figure 4 suggest that TRIM45 might directly interact with p53 to activate p53 signaling. To test this hypothesis, we transfected U87 cells with HA-tagged TRIM45 and Flagtagged p53 expression plasmids. Co-immunoprecipitation (IP) and immunoblot experiments revealed that ectopically expressed TRIM45 interacted with Flag-tagged p53 (Figure 5a). In addition, we found that Flag-tagged TRIM45 interacted with endogenous p53 (Figure 5b). To further verify that TRIM45 interacts with p53 in glioma cells, we performed IP experiments with U87 cell extracts using an anti-p53 antibody or an isotype IgG and found that TRIM45 interacted with p53 under physiological conditions in glioma cells 
a

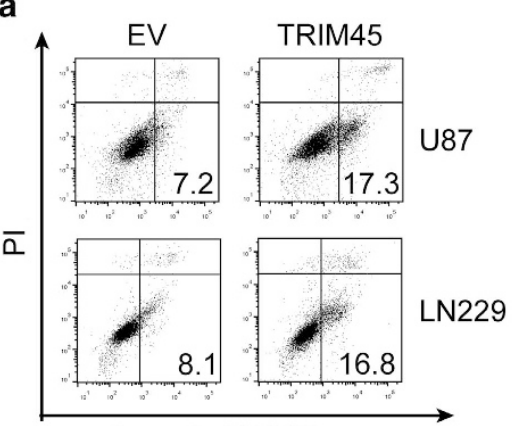

Annexin V-FITC

C

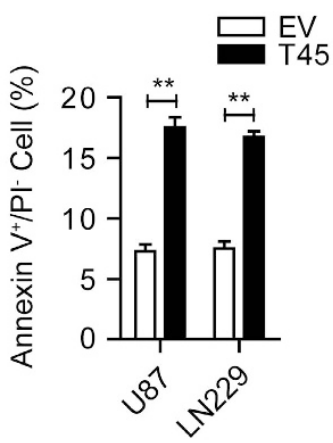

$\alpha$-Caspase 3

(Cleaved) $\alpha-\beta$-actin

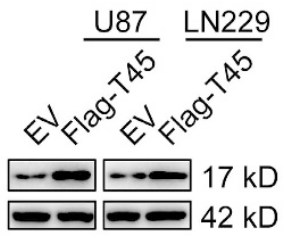

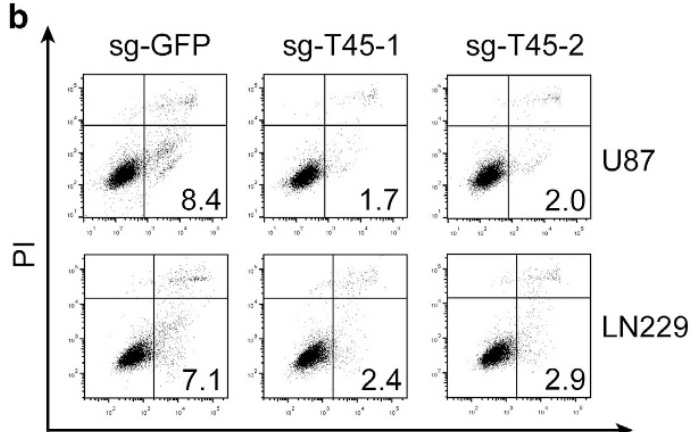

Annexin V-FITC

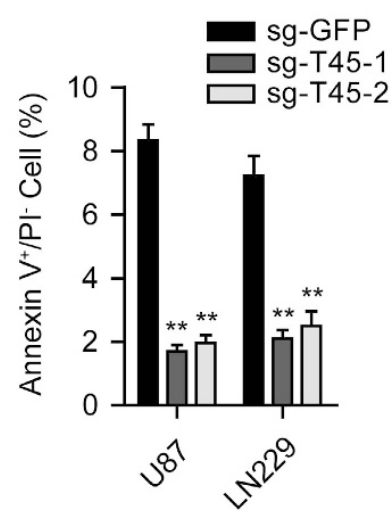

d

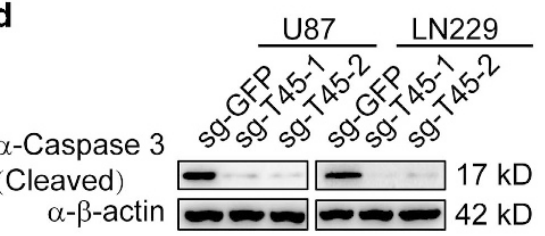

Figure 3 TRIM45 promotes apoptosis in GBM cells. (a and $\mathbf{b}$ ) The TRIM45-overexpressing (a) and KO (b) U87 and LN229 cells were incubated with FITC-Annexin V solution and subjected to FACS analysis. (c and d) Lysates of TRIM45-overexpressing (c) and TRIM45 KO (d) U87 and LN229 cells were immunoblotted with an anti-caspase-3 antibody. $\beta$-Actin was used as a loading control. Each bar represents the mean \pm S.D. of three independent experiments. ${ }^{* \star} P<0.01$

(Figure 5c). Taken together, these results suggest that TRIM45 interacts with p53 in physiological conditions.

To identify the domain of TRIM45 responsible for interaction with p53, we generated a series of deletion mutants of TRIM45 and tested their ability to interact with p53 (Figure 5d, top). p53 exhibited a strong interaction with TRIM45- $\triangle$ RING and TRIM45- $\Delta$ B-box, a weak interaction with TRIM45- $\Delta C C$, and no interaction with TRIM45- $\triangle$ FLMN. These results indicate that the FLMN region is essential for the interaction of TRIM45 with $\mathrm{p} 53$ and that the $\mathrm{CC}$ region might also be required for binding to p53 (Figure 5d, bottom). We also generated three deletion mutants of p53 and evaluated their ability to bind with TRIM45 (Figure 5e, top). We found that TRIM45 exhibited a strong interaction with p53 (100-393) and p53 ( $100-300)$ mutants, but no interaction with p53 (1-300) mutant, indicating that the C-terminal domain of p53 (301-393) is necessary for binding to TRIM45 (Figure 5e, bottom).

TRIM45 mediates the K48-K63 ubiquitination transition of p53 via its E3 ligase activity. As TRIM45 is an E3 ligase, ${ }^{19}$ we next determined whether TRIM45 affects p53 stability via ubiquitination. First, we found that TRIM45 promoted the polyubiquitination of p53 in a dose-dependent manner (Figure 6a). Having demonstrated that TRIM45 is a p53interacting E3 ligase that targets p53 for ubiquitination, we further characterized the specific types of polyubiquitination of p53 by TRIM45. Interestingly, we found that TRIM45 promoted the K63-linked ubiquitination and inhibits the K48-linked ubiquitination of p53, but did not affect other ubiquitination types of p53 (Figure 6b). As a positive control, MDM2 promoted the K48-linked ubiquitination of p53 (Figure $6 \mathrm{~b}$ ). To assess the possible role of MDM2 in TRIM45-mediated effect on p53, we tested whether TRIM45 could affect p53 ubiquitination in MDM2-knockdown U87 cells. We found that MDM2-knockdown significantly enhanced the TRIM45-mediated K63-linked ubiquitination of p53. However, TRIM45 overexpression could not inhibit the K48-linked ubiquitination of p53 in MDM2-knockdown cells (Supplementary Figure 10). We next investigated whether TRIM45 inhibits K48-linked ubiquitination through disrupting the MDM2-p53 complex. The co-IP assay showed that TRIM45 did not affect the interaction between 
a

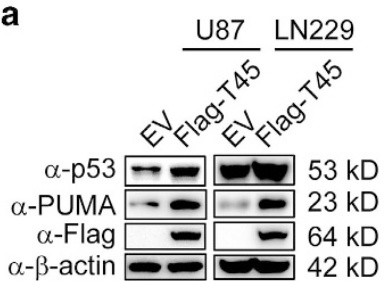

b

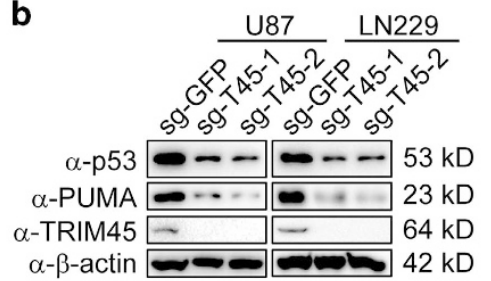

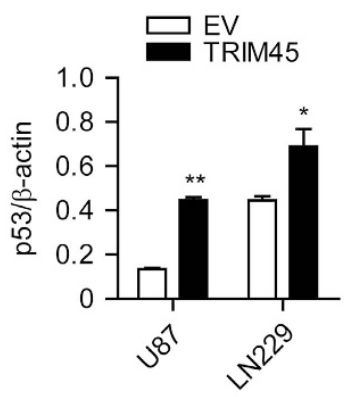

e

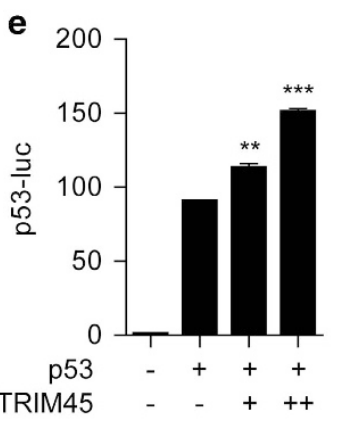

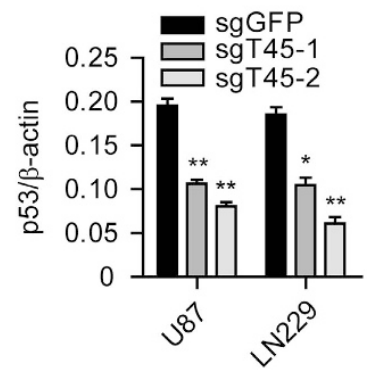

$\mathrm{CHX}(\min ) \quad 0 \quad 153060$ $\alpha$-p53 $\alpha$-TRIM45 - 64 kD sg-GFP $\alpha-\beta$-actin 42 kD $\alpha-p 53 \sim$ $\alpha-$ TRIM45 $\alpha-\beta$-actin $42 \mathrm{kD}$

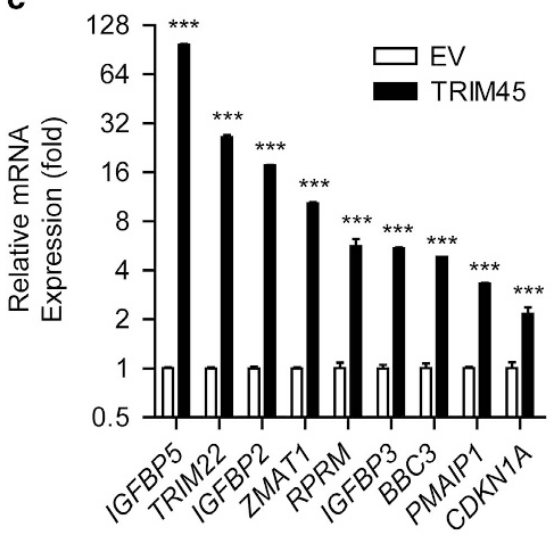

$\begin{array}{ll}\text { d } & 1.5 \\ & \end{array} \begin{aligned} & \square_{\text {sg-GFP }}^{\text {sg-T45-1 }} \\ & \text { sg-T45-2 }\end{aligned}$

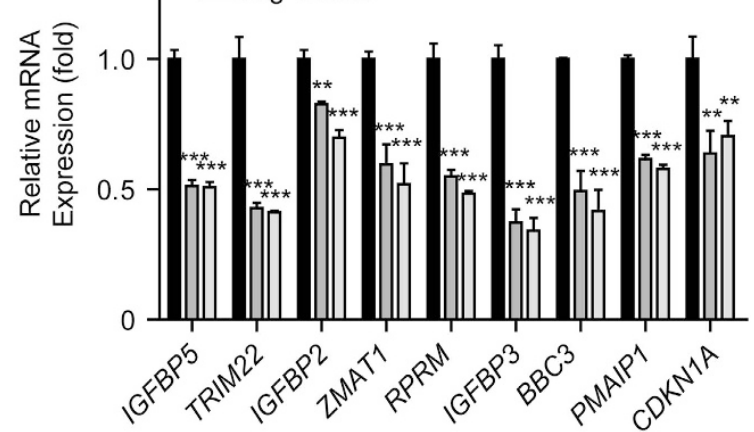

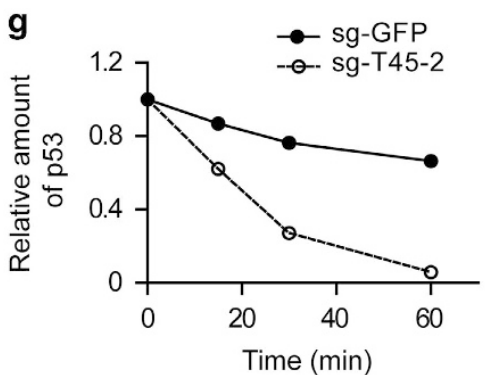

h

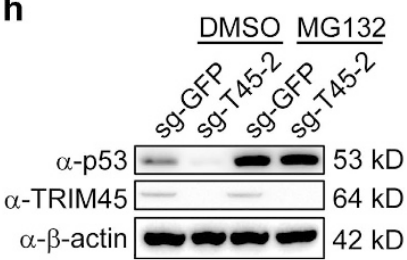

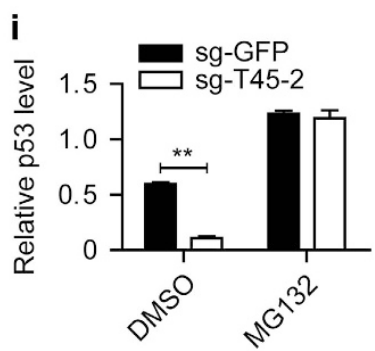

Figure 4 TRIM45 stabilizes and activates p53 in glioma cells. (a) Lysates of TRIM45-overexpressing and control U87 and LN229 cells were immunoblotted with anti-p53, anti-PUMA, and anti-Flag antibodies. $\beta$-Actin was used as a loading control. (b) Lysates of TRIM45 KO and control U87 and LN229 cells were immunoblotted with anti-p53, anti-PUMA, and anti-TRIM45 antibodies. $\beta$-Actin was used as a loading control. (c and d) The mRNA levels of p53 target genes in TRIM45 overexpression and KO U87 cells were analyzed using real-time PCR. (e) Luciferase activity in U87 cells transfected with p53-luc, p53, and an empty vector or a vector expressing TRIM45 (0, 200, and 400 ng). Results are presented as reporter gene activity relative to Renilla luciferase activity. ( $\mathbf{f}$ and $\mathbf{g}$ ) Immunoblot analysis of extracts of TRIM45 KO or control U87 cells treated for various times (as indicated above each lane) with CHX. (h and i) Lysates of TRIM45 KO and control U87 cells treated with MG132 or dimethyl sulfoxide (DMSO) were immunoblotted with antip53 and anti-TRIM45 antibodies. $\beta$-Actin was used as a loading control. Each bar represents the mean \pm S.D. of three independent experiments. ${ }^{*} P<0.05$, ${ }^{\star \star} P<0.01$, and ${ }^{* * *} P<0.001$

MDM2 and p53 (Supplementary Figure 11). Consistent with the results in Figure $6 \mathrm{~b}$, mutation of $\mathrm{K} 48$ to arginine (Ub-K48R) markedly increased its ability to be conjugated to $\mathrm{p} 53$ by TRIM45, while Ub-K63R reduced its ability to be conjugated to p53 by TRIM45 (Supplementary Figure 12). Furthermore, TRIM45 overexpression potentiated the total ubiquitination and K63-linked ubiquitination and inhibited the K48-linked ubiquitination of endogenous p53 (Supplementary Figure 13). These data suggest that TRIM45 mediates the K48-K63 ubiquitination transition of p53.
We next investigated whether TRIM45 mediates the K48K63 ubiquitination transition of p53 via its E3 ligase activity, and found that the TRIM45 mutant C29A that lacks E3 ligase activity failed to promote the K63-linked ubiquitination or inhibit the K48-linked ubiquitination of p53 in the presence of MG132 (Figures $6 \mathrm{c}$ and $\mathrm{d}$ ). In addition, wild-type TRIM45, but not TRIM45 C29A, increased p53 protein levels (Figure 6e). Consistent with this result, TRIM45 C29A did not affect the transcriptional activity of p53 (Figure 6f). Taken together, these results indicate that the E3 ligase activity of TRIM45 is 
a

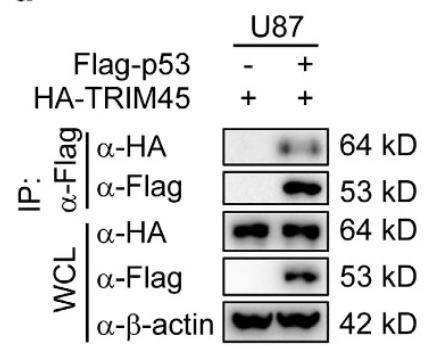

b

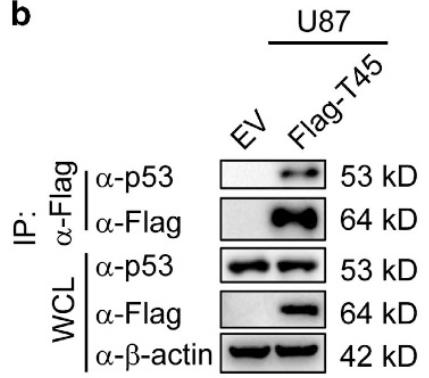

c

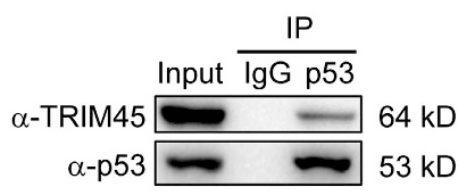

d
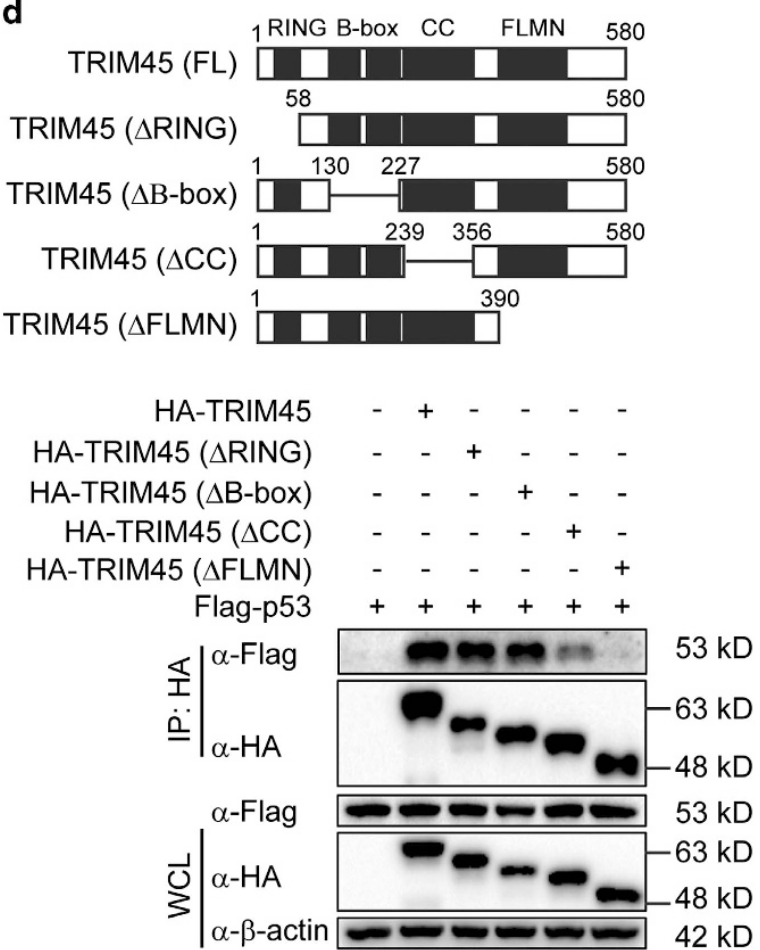

e

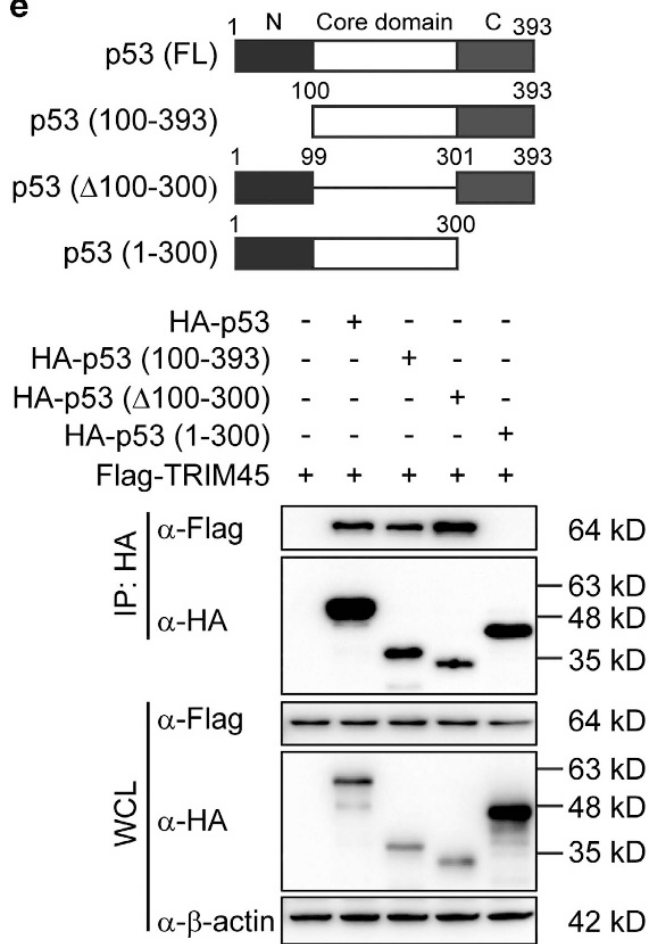

Figure 5 TRIM45 interacts with p53. (a) U87 cells were transfected with Flag-p53 and hemagglutinin (HA)-TRIM45 plasmids. The lysates were immunoprecipitated with antiFlag beads and immunoblotted with anti-HA. (b) Cell extracts of TRIM45-overexpressing or control U87 cells were immunoprecipitated with anti-Flag beads and immunoblotted with the anti-p53 antibody. (c) U87 cell extracts were immunoprecipitated with immunoglobulin G (IgG) or the anti-p53 antibody and immunoblotted together with whole-cell lysates and the anti-TRIM45 antibody. (d) Top: The domain structure of TRIM45. Bottom: 293T cells were transfected with Flag-p53 and HA-TRIM45 or various HA-tagged TRIM45 mutant constructs and treated with $10 \mu \mathrm{M}$ MG132. Whole-cell extracts were immunoprecipitated with anti-HA beads and immunoblotted with anti-Flag antibody. (e) Top: the domain structure of p53. Bottom: 293T cells were transfected with Flag-TRIM45 and HA-p53 or with various HA-tagged p53 mutant constructs, and treated with $10 \mu \mathrm{M}$ MG132. Whole-cell extracts were immunoprecipitated with anti-HA beads and immunoblotted with an anti-Flag antibody

indispensable for the K48-K63 ubiquitination transition of p53 and for p53 stabilization and activation.

TRIM45 stabilizes p53 by catalyzing the K63-linked polyubiquitination of p53 at its C-terminal six lysine residues. K48-linked polyubiquitination has a critical role in p53 degradation, and the major lysine residues that are ubiquitinated by E3 ligases are the six lysines in the C-terminal of p53, including K370, K372, K373, K381, $\mathrm{K} 382$, and $\mathrm{K}_{386}{ }^{23}$ The results presented in Figures $6 \mathrm{~b}-\mathrm{d}$ prompted us to speculate that TRIM45-mediated K63-linked polyubiquitination might compete with K48-linked polyubiquitination at the same lysine residues, thereby protecting p53 from K48-linked polyubiquitination. To test this hypothesis, we constructed a series of Flag-tagged p53 mutants with multiple lysine residues substituted with arginine $(R)$ residues (Figure $6 \mathrm{~g}$ ). We found that the K63-linked ubiquitination mediated by TRIM45 was nearly abolished in the p53 6KR mutant but not in other p53 mutants (Figure $6 \mathrm{~h}$ and Supplementary Figure 14). Consistent with this observation, TRIM45 failed to stabilize p53 6KR, but was still able to stabilize the other p53 mutants (Figure 6i). These data suggest that TRIM45 mainly mediates K63-linked polyubiquitination on the C-terminal six lysine residues of p53, thereby inhibiting the availability of those residues for the subsequent K48-linked polyubiquitination that targets p53 for degradation (Figure 7). 


\section{Discussion}

The p53 tumor suppressor network is frequently disrupted in GBM, and the deregulation of p53 antagonists, such as MDM2 and MDM4 (also known as HDMX and MDMX), is a primary contributor to p53 inactivation in this context. ${ }^{24}$ The MDM2 proteins are dysregulated in many human cancers, and they exert their oncogenic activity predominantly by inhibiting the a

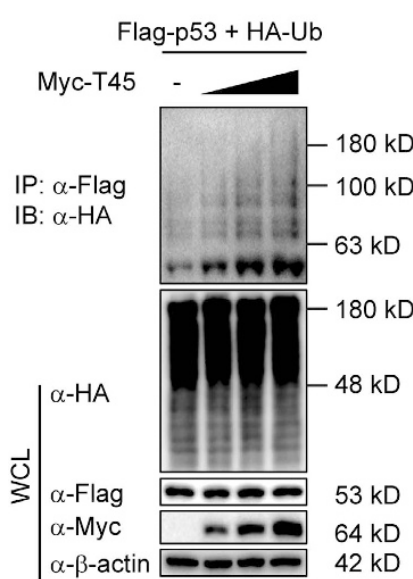

c

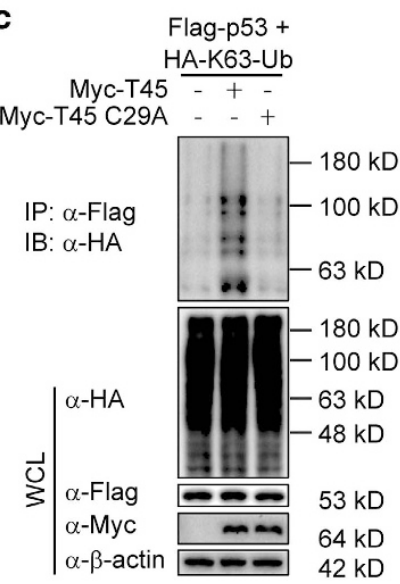

b

Flag-p53

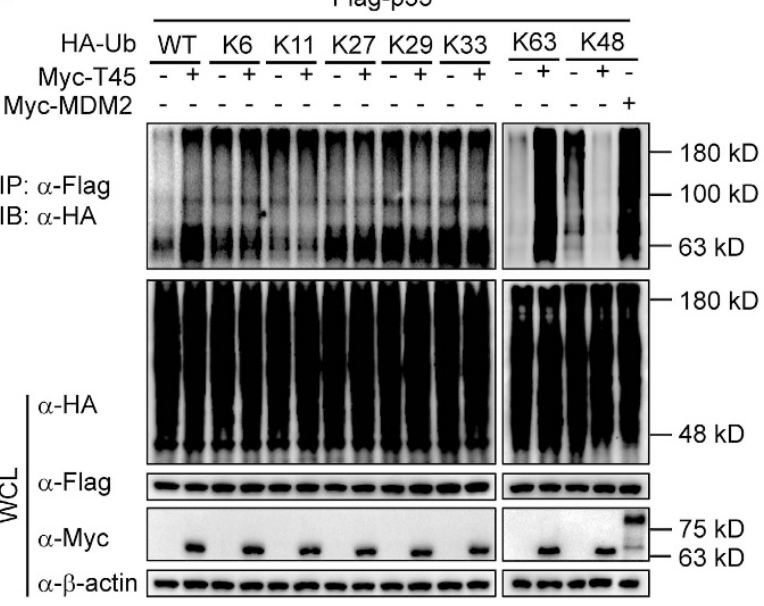

d

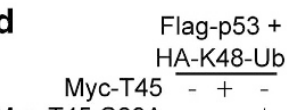

Myc-T45 C29A - -

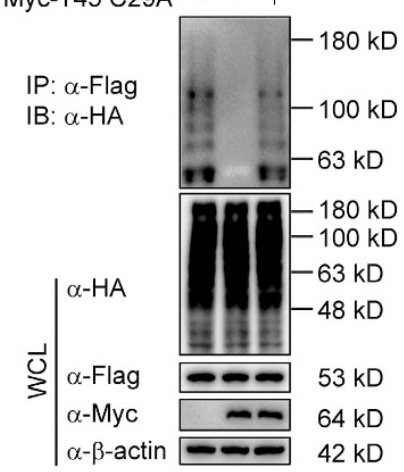

e
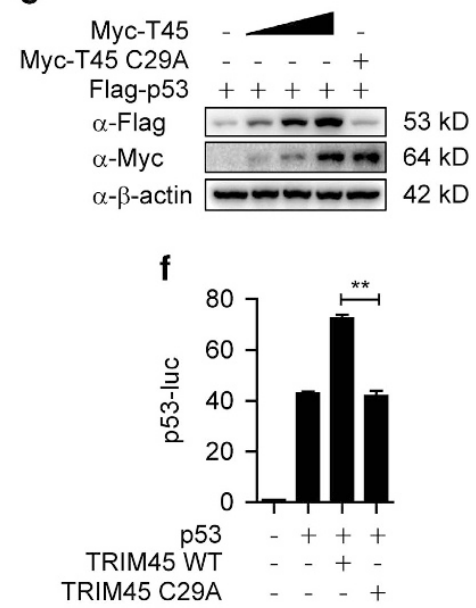

h

Flag-p53

Myc-T45

IP: $\alpha-$ Flag

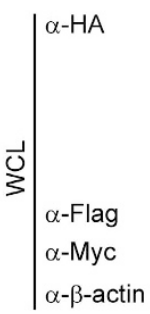

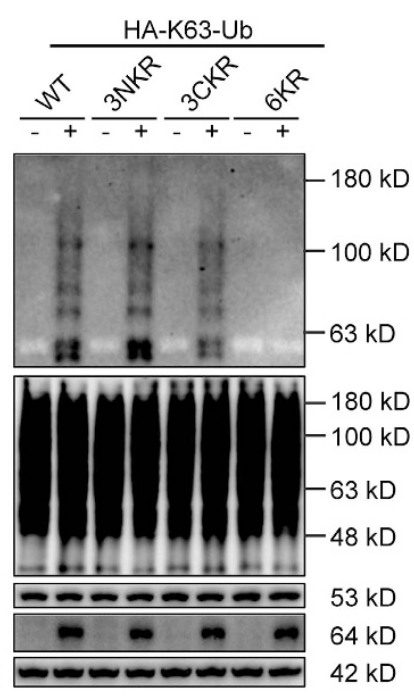

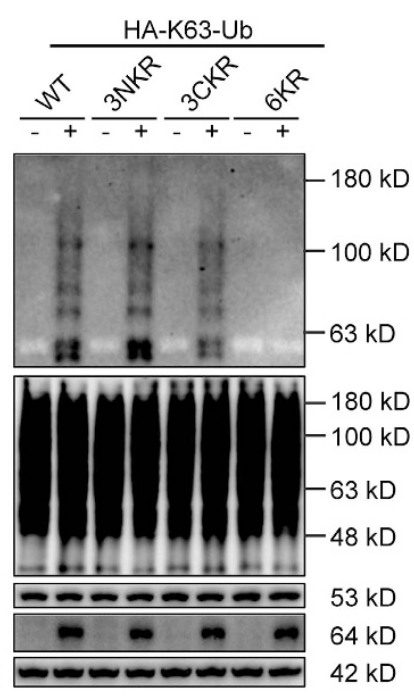

TRIM45 WT

TRIM45 C29A i

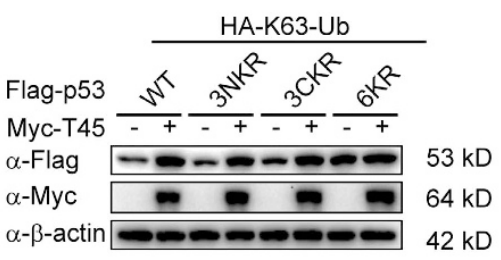


activity of p53. High MDM protein levels can result from gene amplification, increased transcription and aberrant PTMs. ${ }^{24}$ MDM2 is the primary E3 ubiquitin ligase responsible for the conjugation of K48-linked polyubiquitin chains to p53 for its degradation. In addition to MDM2, other molecules, including Pirh2, COP1, and ARF-BP1 also conjugate K48-linked polyubiquitin chains to p53 to target it for proteasomal degradation. ${ }^{25-27}$ These E3 ligases might have redundant functions in regulating p53 degradation.

In contrast to the aforementioned E3 ligases that mediate p53 degradation, several E3 ligases can also stabilize p53 or promote p53 transcriptional activity. WWP1, an HECT domain E3 ligase, induces p53 ubiquitination and stabilizes p53. ${ }^{28}$ Interestingly, Ubc13, a key E2 ubiquitin-conjugating enzyme that induces K63-linked ubiquitination, also promotes K63-linked ubiquitination of p53, and protects p53 from degradation. $^{29}$ In the current study, we found that TRIM45 regulates p53 stability using a similar mechanism. TRIM45 can promote K63-linked polyubiquitination and inhibit K48linked polyubiquitination of p53 (Figure 6b). It is tempting to speculate that TRIM45 acts in conjunction with the E2 ligase Ubc13 to conjugate K63-linked polyubiquitin chains to p53.

Polyubiquitination of p53 catalyzed by TRIM45 was mapped to C-terminal six lysine residues (Figure $6 \mathrm{~h}$ and Supplementary Figure S8), which are also targeted by MDM2 for K48-linked polyubiquitination. ${ }^{30,31}$ These findings suggest that the polyubiquitin chains of distinct linkages may compete for the same residues of p53. Different modifications by different regulators are known to compete with each other for the same site in some proteins. Direct competition between acetylation and ubiquitination for the same C-terminal lysine residues of p53 has been reported to stabilize and activate p53. ${ }^{32,33}$ It has also been reported that the competition between $\mathrm{K} 11$ - and K48-linked polyubiquitination stabilizes a critical antiviral regulator, STING. ${ }^{34}$

Taken together, these data support a model in which TRIM45 functions as a tumor suppressor by stabilizing p53 (Figure 7). In normal cells, p53 protein amount is tightly controlled by MDM2, or other E3 ligases that catalyze the K48linked polyubiquitination of p53 for proteasome-dependent
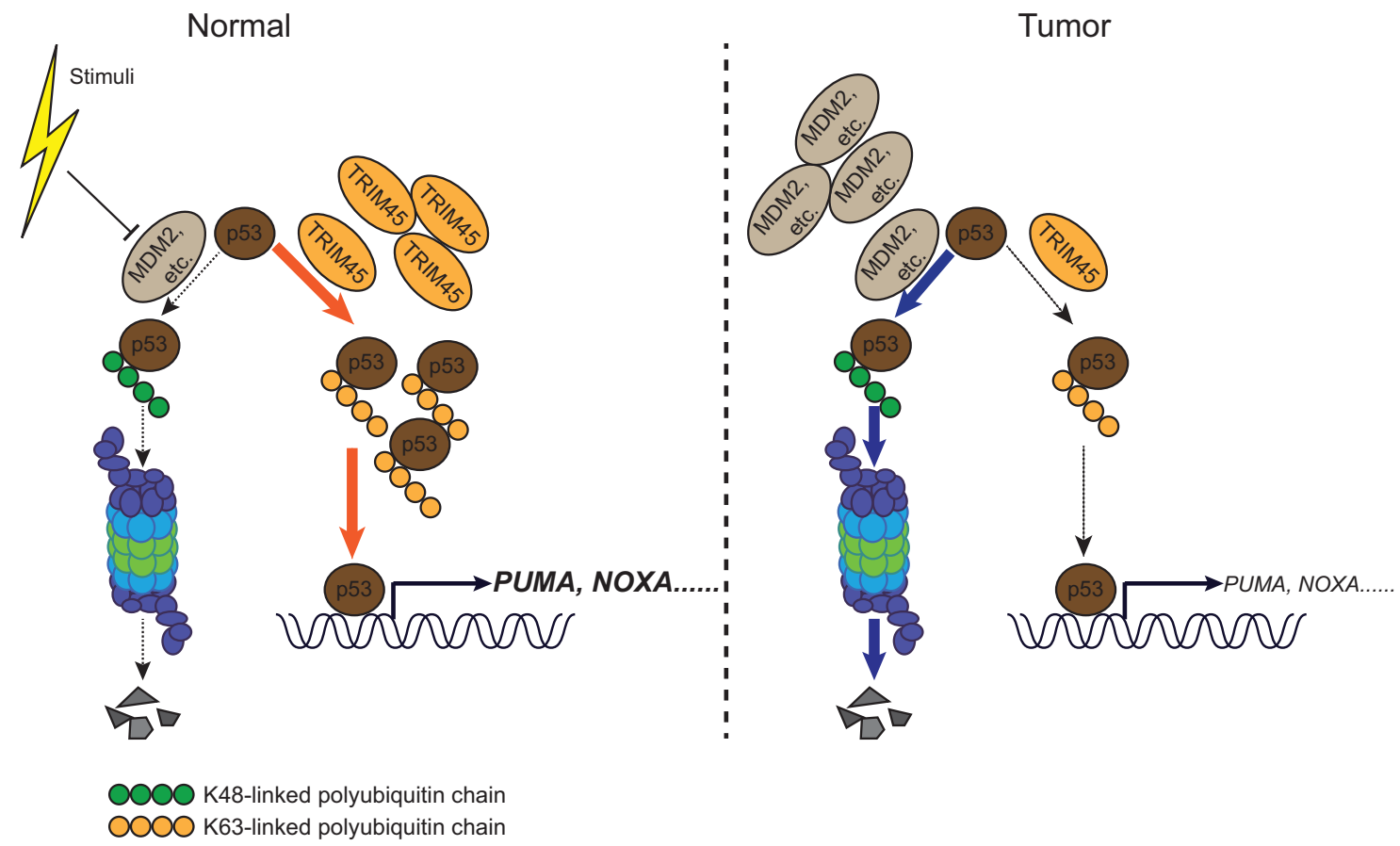

Figure 7 A proposed model illustrating the mechanism by which TRIM45 regulates p53 stability

\footnotetext{
Figure 6 TRIM45 mediates the K48-K63 ubiquitination transition of p53. (a) Lysates of U87 cells transfected with plasmids expressing Flag-p53, hemagglutinin (HA)-ubiquitin and increasing amounts of Myc-TIRM45 and treated with MG132 were immunoprecipitated with anti-Flag beads and immunoblotted with an anti-HA antibody. (b) U87 cells were transfected with plasmids expressing Flag-p53, Myc-TIRM45 or Myc-MDM2 together with HA-ubiquitin or its indicated mutants in the presence of MG132. The cell lysates were immunoprecipitated with anti-Flag beads and immunoblotted with an anti-HA antibody. (c and d) Lysates of U87 cells transfected with plasmids expressing Flag-p53, HA-tagged K63-linked ubiquitin (HA-K63-Ub) (c) or HA-K48-Ub, (d) together with Myc-TIRM45 or Myc-TIRM45 C29A and treated with MG132 were immunoprecipitated with anti-Flag beads and immunoblotted with anti-HA antibody. (e) Immunoblot analysis of extracts of U87 cells transfected with Flag-p53 and increasing amounts of HA-TRIM45 or HA-TRIM45 CA (f) Luciferase activity in U87 cells transfected with p53-luc, p53, and Myc-TIRM45 or Myc-TRIM45 C29A. Results are presented as Firefly luciferase activity relative to Renilla luciferase activity. Each bar represents the mean \pm S.D. of three independent experiments. ${ }^{*} P<0.01$. (g) A schematic diagram of p53 mutants. (h) Lysates of U87 cells transfected with plasmids expressing HA-K63-Ub and wild-type (WT) p53 or p53 mutant together with Myc-TRIM45 or empty vector and treated with MG132 were immunoprecipitated with anti-Flag beads and immunoblotted with an anti-HA antibody. (i) Immunoblot analysis of extracts of U87 cells transfected with plasmids expressing HA-K63-Ub and WT p53 or p53 mutant together with Myc-TRIM45 or empty vector
} 
degradation. Meanwhile, TRIM45 can compete with MDM2 or other E3 ligases to promote the K63-linked polyubiquitination of $\mathrm{p53}$, thereby preventing the K48-linked polyubiquitination of p53 and protecting it from degradation. This process may let cells undergo apoptosis under harmful stresses. In glioma tissues, TRIM45 is downregulated and cannot maintain the sufficient amount of p53 to induce the apoptosis of tumor cells. ${ }^{35}$ Our current findings highlight a previously undescribed mechanism for the regulation of p53 stability.

In summary, we have demonstrated that TRIM45 promotes the K63-linked ubiquitination of p53 to protect p53 from degradation and inactivation, thereby suppressing GBM proliferation and tumorigenicity. These results suggest that TRIM45 acts as a tumor suppressor in malignant glioma. A more comprehensive understanding of the role TRIM45 has in the pathogenesis of malignant glioma may provide an opportunity to develop a novel therapeutic strategy by restoration of TRIM45 expression.

\begin{abstract}
Materials and Methods
Clinical specimens. The samples were obtained from 40 glioma patients who had undergone neurosurgery between 2008 and 2013 at the Third Affiliated Hospital of Soochow University. All samples were obtained from newly diagnosed patients with histologically confirmed primary gliomas. The pathological diagnosis and grading of glioma were assigned according to the WHO classification system. The normal brain tissues were obtained from individuals who had died in traffic accidents and were confirmed to be free of any prior pathologically detectable conditions. Taken together, the collection of samples used in this study comprised normal brain tissue $(n=10)$, pilocytic astrocytoma (WHO grade I; $n=4)$, diffuse astrocytoma (WHO grade II; $n=7$ ), anaplastic astrocytoma (WHO grade III; $n=12$ ), and GBM (WHO grade IV; $n=17$ ) tissues. The study was approved by the Research Ethics Board of the Third Affiliated Hospital of Soochow University, Informed consent was obtained from all individual participants included in the study.
\end{abstract}

Cell culture and transfection. U87 and LN229 cells were cultured in DMEM (Life Technologies, Beijing, China) supplemented with 10\% fetal bovine serum (Gibco, Grand Island, NY, USA) and $1 \mathrm{mM}$ glutamine (Life Technologies). For the gene knockdown and KO assays, cells were infected with lentivirus encoding shRNA or sgRNA, respectively. For the gene overexpression assays, cells were transfected with lentivirus encoding the TRIM45 coding sequence.

Plasmids and antibodies. The TRIM45 expression plasmid was constructed using the GATEWAY System (Life Technologies, Carlsbad, CA, USA). The HAtagged p53 expression plasmid and the p53 domain deletion mutants were kindly provided by Dr. Tiebang Kang (Sun Yat-sen University, Guangzhou, China). All mutants were generated using standard molecular techniques. The anti-TRIM45 antibody (NBP1-53109) was purchased from Novus (Littleton, CO, USA) and antip53 (sc-126) was obtained from Santa Cruz (Santa Cruz, CA, USA). Horseradish peroxidase (HRP)-anti-Flag (M2) (A8592) and anti- $\beta$-actin (A1978) were purchased from Sigma (St. Louis, MO, USA). HRP-anti-hemagglutinin (12013819001) and antic-Myc-HRP (11814150001) were purchased from Roche Applied Science (Mannheim, Germany). The anti-caspase-3 (25546-1-AP) was purchased from Proteintech (Wuhan, Hubei, P.R.C). The anti-MDM2 (YT5186) was purchased from ImmunoWay (Newark, DE, USA). The anti-K63-linked ubiquitin antibody (05-1308) was obtained from Millipore (Billerica, MA, USA). The anti-phospho-Akt (no. 4060), anti-Akt (no. 4691), anti-phospho-JNK (no. 9251), anti-JNK (no. 9252), antiphospho-ERK (no. 9101), anti-ERK (no. 9102), anti-phospho-p38 (no. 9211), antip38 (no. 9212), anti-Puma (no. 4976), and anti-K48-linked ubiquitin (no. 4289) antibodies were obtained from Cell Signaling Technology (Danvers, MA, USA).

Generation of TRIM45 KO cells by CRISPR/Cas9 technology. CRISPR/Cas9 system-mediated KO was performed as described previously, ${ }^{36}$ and the sequence of the target GFP or TRIM45-gRNA are as follows: GFP sgRNA, 5'-GGGCGAGGAGCTGTTCACCG-3', TRIM45 sgRNA 1, 5'-GATGCTGGAGAGCC TACGTG-3', and TRIM45 sgRNA 2, 5'-GTGTGACCTGTGCAACGACA-3'.
IP and immunoblot analysis. Cells were extracted in ice-cold low-salt lysis buffer (50 mM HEPES, $150 \mathrm{mM} \mathrm{NaCl}, 1 \mathrm{mM}$ EDTA, $1.5 \mathrm{mM} \mathrm{MgCl} 2,10 \%$ glycerol, $1 \%$ Triton X-100) supplemented with $5 \mathrm{mg} / \mathrm{ml}$ protease inhibitor cocktail (Roche). Protein concentration was measured with BCA Protein Assay Kit (Pierce, Rockford, IL, USA). For IP experiments, whole-cell extracts were prepared after transfection, followed by incubation overnight with the appropriate antibodies and Protein A/G beads (Thermo, Rockford, IL, USA) or anti-Flag/anti-HA beads (Sigma-Aldrich, St. louis, MO, USA). Beads were washed three times with low-salt lysis buffer. Immunoprecipitates were eluted with $3 \times$ SDS Loading Buffer and resolved by SDSPAGE. Proteins were transferred to PVDF membranes (Bio-Rad, Hercules, CA, USA) and incubated with the appropriate antibodies. For the ubiquitin assays, cell lysates were first immunoprecipitated with anti-Flag beads, then boiled in the presence of $1 \%$ SDS, followed by a second IP with anti-Flag, so that only polyubiquitination modification would be detected by immunoblot analysis with antihemagglutinin.

Luciferase assay. U87 cells were plated in 24-well plates and transfected with plasmids expressing p53-luc (50 ng) and TK-Renilla-luc (10 ng), together with $200 \mathrm{ng}$ Flag-tagged p53 and increasing amounts of TRIM45 using Lipofectamine 2000 (Life Technologies). An empty pcDNA3.1 vector was used to maintain equal amounts of DNA between wells. Cells were harvested $24 \mathrm{~h}$ post transfection, and luciferase activities were measured with Dual-luciferase Assay Kits (Promega, Madison, WI, USA) according to the manufacturer's protocol. Reporter gene activity was determined by normalizing Firefly luciferase to Renilla luciferase activity.

Cell proliferation and anchorage-independent growth assays. Cell proliferation was measured by directly counting the number of the cells. Briefly, triplicate plates of cells were trypsinized and stained with Trypan blue, and unstained cells were counted using a hemocytometer. Anchorage-independent growth assays were performed in 6-well plates. Cell were seeded at a density of $1 \times 10^{4}$ cells per well in DMEM+10\% FBS containing $0.35 \%$ low-melting agarose on top of the agar layer containing $0.5 \%$ low-melting agarose DMEM+10\% FBS. Colonies were counted 14-21 days after seeding.

Xenograft tumor formation in mice. Xenograft tumor formation assays were conducted as described previously. ${ }^{37}$ Briefly, BALB/c-nude mice (4-week-old) were purchased from Guangdong Medical Laboratory Animal Center. All experimental procedures were approved by the Institutional Animal Care and Use Committee of the Sun Yat-sen University. The mice were subcutaneously inoculated with $5 \times 10^{6}$ of the indicated cells. Tumor growth was monitored every five days. Tumor volume was calculated using the formula: $V=0.5 \times$ length $\times$ width $\times$ width. The tumor-bearing mice were killed 40 days after inoculation, and the tumors were subsequently removed for further study.

Annexin V-binding assay. The FITC-Annexin V Apoptosis Detection Kit I (BD Bioscience, Franklin Lakes, NJ, USA) was used to quantify apoptotic cells according to the manufacturer's instructions.

Statistical analysis. The data are represented as the mean \pm S.D. when indicated, and the Student's $t$-test was used for all statistical analyses using the GraphPad Prism 5.0 software (GraphPad Software, Inc., La Jolla, CA, USA). Differences between groups were considered statistically significant when the $P$ value was $<0.05$.

\section{Conflict of Interest}

The authors declare no conflict of interest.

Acknowledgements. This work was supported by National Natural Science Foundation of China (91629101 and 31522018), National Key Basic Research Program of China (2015CB859800, 2014CB910800 and 2014CB745203), Guangdong Natural Science Funds for Distinguished Young Scholar (S2013050014772), Guangdong Innovative Research Team Program (Nos 2011Y035 and 201001Y0104687244), the Fundamental Research Funds for the Central Universities (15lgjc02), and the Training Program for Outstanding Young Teachers in Higher Education institutions of Guangdong Province (YQ2015001). R-FW was, in part, supported by grants (CA101795 and DA030338) from $\mathrm{NCl}$ and NIDA, NIH. FZ is partially supported by the National Natural Science Foundation of 
China (81302197), Jiangsu Provincial Special Programe of Medical Science (BL2014035), Changzhou Science and Technology Support Program (CE20165048), Changzhou High-Level Medical Talents Training Project (2016CZBJ006), and Changzhou Municipal Commisions of Health and Family Planning Major Scientific and Technological Project (ZD201620).

1. Gladson CL, Prayson RA, Liu WM. The pathobiology of glioma tumors. Annu Rev Pathol 2010; 5: 33-50.

2. Huse JT, Holland EC. Targeting brain cancer: advances in the molecular pathology of malignant glioma and medulloblastoma. Nat Rev Cancer. 2010; 10: 319-331.

3. Dolecek TA, Propp JM, Stroup NE, Kruchko C. CBTRUS statistical report: primary brain and central nervous system tumors diagnosed in the United States in 2005-2009. Neurooncology 2012; 14(Suppl 5): v1-49.

4. Van Meir EG, Hadjipanayis CG, Norden AD, Shu HK, Wen PY, Olson JJ. Exciting new advances in neuro-oncology: the avenue to a cure for malignant glioma. CA Cancer J Clin 2010; 60: 166-193.

5. Wen PY, Kesari S. Malignant gliomas in adults. N Engl J Med 2008; 359: 492-507.

6. Bieging KT, Mello SS, Attardi LD. Unravelling mechanisms of p53-mediated tumour suppression. Nat Rev Cancer 2014; 14: 359-370.

7. Bode AM, Dong Z. Post-translational modification of p53 in tumorigenesis. Nat Rev Cancer 2004; 4: 793-805

8. Dai C, Gu W. P53 post-translational modification: deregulated in tumorigenesis. Trends Mol Med 2010; 16: 528-536.

9. Ashcroft M, Vousden KH. Regulation of p53 stability. Oncogene 1999; 18: 7637-7643.

10. Hershko A, Ciechanover A. The ubiquitin system. Annu Rev Biochem 1998; 67: 425-479.

11. Husnjak K, Dikic I. Ubiquitin-binding proteins: decoders of ubiquitin-mediated cellular functions. Annu Rev Biochem 2012; 81: 291-322.

12. Lee JT, Gu W. The multiple levels of regulation by p53 ubiquitination. Cell Death Differ 2010; 17: 86-92.

13. Haupt $Y$, Maya R, Kazaz A, Oren M. Mdm2 promotes the rapid degradation of p53. Nature 1997; 387: 296-299.

14. Kubbutat MH, Jones SN, Vousden KH. Regulation of p53 stability by Mdm2. Nature 1997; 387: 299-303.

15. Saurin AJ, Borden KL, Boddy MN, Freemont PS. Does this have a familiar RING? Trends Biochem Sci 1996; 21: 208-214.

16. Reymond A, Meroni G, Fantozzi A, Merla G, Cairo S, Luzi L et al. The tripartite motif family identifies cell compartments. EMBO J 2001; 20: 2140-2151.

17. Ozato K, Shin DM, Chang TH, Morse HC III. TRIM family proteins and their emerging roles in innate immunity. Nat Rev Immunol 2008; 8: 849-860.

18. Wang Y, Li Y, Qi X, Yuan W, Ai J, Zhu C et al. TRIM45, a novel human RBCC/TRIM protein, inhibits transcriptional activities of ElK-1 and AP-1. Biochem Biophys Res Commun 2004; 323: 9-16.

19. Sato T, Takahashi H, Hatakeyama S, Iguchi A, Ariga T. The TRIM-FLMN protein TRIM45 directly interacts with RACK1 and negatively regulates PKC-mediated signaling pathway. Oncogene 2015; 34: 1280-1291.

20. Shibata M, Sato T, Nukiwa R, Ariga T, Hatakeyama S. TRIM45 negatively regulates NF-kappaB-mediated transcription and suppresses cell proliferation. Biochem Biophys Res Commun 2012; 423: 104-109.

21. Cancer Genome Atlas Research N. Comprehensive genomic characterization defines human glioblastoma genes and core pathways. Nature 2008; 455: 1061-1068.
22. Parsons DW, Jones S, Zhang X, Lin JC, Leary RJ, Angenendt $P$ et al. An integrated genomic analysis of human glioblastoma multiforme. Science 2008; 321: 1807-1812.

23. Hock A, Vousden $\mathrm{KH}$. Regulation of the p53 pathway by ubiquitin and related proteins. Int $\mathrm{J}$ Biochem Cell Biol 2010; 42: 1618-1621.

24. Wade M, Li YC, Wahl GM. MDM2, MDMX and p53 in oncogenesis and cancer therapy. Nat Rev Cancer 2013; 13: 83-96.

25. Leng RP, Lin Y, Ma W, Wu H, Lemmers B, Chung S et al. Pirh2, a p53-induced ubiquitinprotein ligase, promotes p53 degradation. Cell 2003; 112: 779-791.

26. Dornan D, Wertz I, Shimizu H, Arnott D, Frantz GD, Dowd P et al. The ubiquitin ligase COP1 is a critical negative regulator of p53. Nature 2004; 429: 86-92.

27. Chen D, Kon N, Li M, Zhang W, Qin J, Gu W. ARF-BP1/Mule is a critical mediator of the ARF tumor suppressor. Cell 2005; 121: 1071-1083.

28. Laine A, Ronai Z. Regulation of $p 53$ localization and transcription by the HECT domain E3 ligase WWP1. Oncogene 2007; 26: 1477-1483.

29. Laine A, Topisirovic I, Zhai D, Reed JC, Borden KL, Ronai Z. Regulation of p53 localization and activity by Ubc13. Mol Cell Biol 2006; 26: 8901-8913.

30. Kubbutat MH, Ludwig RL, Ashcroft M, Vousden KH. Regulation of Mdm2-directed degradation by the $C$ terminus of p53. Mol Cell Biol 1998; 18: 5690-5698.

31. Rodriguez MS, Desterro JM, Lain S, Lane DP, Hay RT. Multiple C-terminal lysine residues target p53 for ubiquitin-proteasome-mediated degradation. Mol Cell Biol 2000; 20 $8458-8467$.

32. Ito A, Kawaguchi Y, Lai CH, Kovacs JJ, Higashimoto Y, Appella E et al. MDM2-HDAC1mediated deacetylation of $\mathrm{p} 53$ is required for its degradation. EMBO $J 2002 ; 21$ $6236-6245$.

33. Li M, Luo J, Brooks CL, Gu W. Acetylation of p53 inhibits its ubiquitination by Mdm2. J Biol Chem 2002; 277: 50607-50611.

34. Qin Y, Zhou MT, Hu MM, Hu YH, Zhang J, Guo L et al. RNF26 temporally regulates virustriggered type I interferon induction by two distinct mechanisms. PLOS Pathog 2014; 10 : e1004358.

35. Symonds H, Krall L, Remington L, Saenz-Robles M, Lowe S, Jacks T et al. P53-dependent apoptosis suppresses tumor growth and progression in vivo. Cell 1994; 78: 703-711.

36. Shalem O, Sanjana NE, Hartenian E, Shi X, Scott DA, Mikkelsen TS et al. Genome-scale CRISPR-Cas9 knockout screening in human cells. Science 2014; 343: 84-87.

37. Zhang J, Zhang P, Wei Y, Piao HL, Wang W, Maddika S et al. Deubiquitylation and stabilization of PTEN by USP13. Nat Cell Biol 2013; 15: 1486-1494.

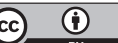

Cell Death and Disease is an open-access journal published by Nature Publishing Group. This work is licensed under a Creative Commons Attribution 4.0 International License. The images or other third party material in this article are included in the article's Creative Commons license, unless indicated otherwise in the credit line; if the material is not included under the Creative Commons license, users will need to obtain permission from the license holder to reproduce the material. To view a copy of this license, visit http://creativecommons.org/licenses/by/4.0/

(C) The Author(s) 2017

Supplementary Information accompanies this paper on Cell Death and Disease website (http://www.nature.com/cddis) 